Trauma Berufskrankh 2009 · 11 [Suppl 2]:

222-228

DOI 10.1007/s10039-008-1445-2

Online publiziert: 17. Oktober 2008

(c) Springer Medizin Verlag 2008

\author{
M. Diefenbeck ${ }^{1} \cdot$ R. Beickert ${ }^{2} \cdot$ G.O. Hofmann ${ }^{1,3}$ \\ ${ }^{1}$ Klinik für Unfall-, Hand- und Wiederherstellungschirurgie, \\ Friedrich-Schiller-Universität Jena \\ ${ }^{2}$ BG-Unfallklinik Murnau \\ ${ }^{3}$ Klinik für Unfall- und Wiederherstellungschirurgie, \\ BG-Kliniken Bergmannstrost, Halle/Saale
}

\title{
Begutachtung der Osteitis
}

Die Osteitis ist die Infektion eines Knochens durch Mikroorganismen, meist Bakterien, als Folge einer Verletzung oder Operation. Sie ist von der hämatogenen Osteomyelitis zu unterscheiden, bei der Bakterien über das Gefäßsystem von einem meist okkulten Herd aus ohne Trauma in den Markraum des Knochens (Myelitis) gestreut werden.

Unterteilt wird die Osteitis in eine akute und eine chronische Form. Die akute postoperative Osteitis tritt innerhalb von 4 Wochen nach dem Unfall und der operativen Frakturversorgung auf [7]. Alle Infekte nach diesem Zeitraum werden als chronische Osteitiden bezeichnet. Bei protrahiertem Verlauf geht die akute Osteitis in die chronische über. Die Begutachtung wird fast ausschließlich bei der chronischen Osteitis als Folge einer Verletzung notwendig.

\section{Inzidenz}

Die Häufigkeit einer akuten postoperativen Osteitis wird in der Literatur für Elektiveingriffe mit $0,1-1,7 \%$ und für die operative Versorgung von geschlossenen Frakturen mit $1-8,1 \%$ angegeben $[3,5]$. Bei der Behandlung offener Frakturen steigt die Rate auf 2,7-43\% [4].

Die Inzidenz der chronischen Osteitis dagegen gilt als ,eines der am besten gehüteten Geheimnisse“ der Chirurgie [10]. Dies hat viele verschiedene Gründe, die von Definitionsproblemen über methodische Schwierigkeiten, fehlende prospektive Studien, mangelhafte retrospektive Analysen bis zu Verdrängungsmechanismen der behandelnden Ärzte reichen. Wie viele der oben erwähnten akuten
Osteitiden in chronische Formen übergehen, ist ebenfalls nicht bekannt.

Für die Prognose - und damit auch für die Begutachtung - gilt weiterhin, dass die chronische Osteitis niemals heilt, sondern entweder „ruht“ oder „exazerbiert/erneut aufflammt“ $[12,15]$. Durch zunehmende Radikalität der chirurgischen Maßnahmen und verbesserte Möglichkeiten der Überbrückung von Knochen- und Weichteildefekten könnte diese Aussage in den nächsten Jahren revidiert werden. Allerdings müssen bei radikaleren Therapieformen größere operative Eingriffe und längere Behandlungszeiten in Kauf genommen werden, die möglicherweise zu einer Funktionsverschlechterung angrenzender Gelenke führen, was für die gutachterliche Beurteilung von Bedeutung ist.

\section{Rechtliche Grundlagen}

Begutachtung ist in den meisten Rechtsbereichen eine Momentaufnahme, eine Beschreibung des Istzustands. Nur im Haftpflichtrecht wird die Frage nach der Zukunftsprognose gestellt, weil hier der materielle und der immaterielle Schaden abgefunden werden können. In der privaten Unfallversicherung muss der Schaden spätestens zum Ende des 3. Jahrs nach dem Unfall entschädigt werden, wobei auch hier nur der Istzustand in die Einschätzung eingeht, nicht jedoch das $\mathrm{Zu}$ kunftsrisiko.

Im Rechtsbereich der Gesetzlichen Unfallversicherung und im Sozialen Entschädigungsrecht sind die Möglichkeiten der Verschlimmerung und der Besserung eingebaut, sodass die Höhe von MdE
(Minderung der Erwerbsfähigkeit) bzw. GdB (Grad der Behinderung) den aktuellen Befunden angepasst werden kann.

Das Vorhandensein einer chronischen Osteitis wird nur im Sozialen Entschädigungsrecht (Bundesversorgungsrecht u. a.) gewürdigt. In der Gesetzlichen Unfallversicherung ist dagegen kein „Osteitiszuschlag“"vorgesehen. Diese Auffassung beruht auf der Feststellung der Sozialgerichtsbarkeit, Rentenbegutachtung sei im Kern Funktionsbegutachtung. Maßgeblich für die Höhe der MdE ist die Beeinträchtigung des körperlichen und geistigen Leistungsvermögens, wobei es nur auf die gegenwärtige Einbuße ankomme. $\mathrm{Zu}$ künftige, ggf. aufgrund gesicherter Erfahrungen absehbare Schäden können nicht berücksichtigt werden [Bundessozialgericht (BSG), SozR $2200 \$ 581$ Nr. 6] [16].

\section{Krankheitsbilder der Osteitis und Kausalitätsfragen}

\section{Akute Osteitis}

Bei ihr treten innerhalb von 4 Wochen nach der operativen Versorgung Symptome wie Rötung, Schwellung, Überwärmung und Schmerzhaftigkeit der Operationswunde auf. Meist besteht eine deutliche Erhöhung der Infektparameter im Labor; klinisch können Fieber und ein allgemeines Krankheitsgefühl vorliegen.

Therapeutisch muss dringlich eine operative Revision durchgeführt werden. Dabei entleert sich meist eitriges Sekret. Der Operationssitus wird débridiert, gespült, ggf. werden lokale Antibiotikaträger und/oder Drainagen eingelegt und die Wunde je nach Befund temporär oder 
definitiv verschlossen. Häufig sind weitere Revisionen bis hin zur Entfernung des einliegenden Osteosynthesematerials und eine äußerer Stabilisierung mittels Fixateur externe notwendig.

Wichtig für die Begutachtung ist, dass hierdurch der Behandlungsverlauf verlängert und erschwert wird. Längere Bettruhe kann z. B. zu Muskelatrophie und Inaktivitätsosteoporose beitragen; die Ruhigstellung von angrenzenden Gelenken durch Schienen oder Fixateur externe Bewegungseinschränkungen und Kontrakturen verursachen. Alle diese Funktionseinschränkungen sind später bei einer Begutachtung zu berücksichtigen, falls sie sich nicht vollständig zurückgebildet haben. Allgemein ist also von einer schlechteren Funktion nach durchgemachter akuter Osteitis im Vergleich zu einem regelrechten Heilverlauf auszugehen, auch wenn zum Zeitpunkt der Begutachtung der Infekt beruhig ist.

Falls die Infektberuhigung der akuten Osteitis in einem angemessenen Zeitraum nicht gelingt, geht sie in eine chronische Form über. Diese ist meist durch das Auftreten von Knochennekrosen (Sequestern) und Fisteln gekennzeichnet und wird unter „Chronische Osteitis“ abgehandelt.

\section{Kausalität}

Kausalitätsfragen sind bei der akuten Osteitis selten, da der zeitliche Zusammenhang gewahrt ist. Fragen nach dem Zusammenhang treten meist nur dann auf, wenn primär keine Knochenbruchverletzung vorgelegen hat. Aber auch eine Weichteilquetschung kann zu einer akuten Osteitis führen. Gefordert wird die erhebliche Einwirkung, also die Quetschung von Weichteilstrukturen mit nachfolgender Hämatombildung oder Nekrotisierung von Haut- und Unterhautfettgewebe. Durch die Weichteilverletzung besteht bei örtlich herabgesetzter Abwehrlage eine Eintrittspforte für Keime, die sich absiedeln, vermehren und den Knochen befallen können. Dies gilt auch bei Herabsetzung der allgemeinen Widerstandskraft, z. B. beim Mehrfachverletzten, beim Multimorbiden oder beim Vorliegen von Begleiterkrankungen wie Diabetes mellitus und periphere arterielle Verschlusskrankheit (pAVK). So kann beim Diabe- tespatienten eine Quetschung des Fußes zur Infektion der Weichteile mit nachfolgender Osteitis bis hin zum Gliedmaßenverlust führen. Die Verletzung bleibt in diesem Fall, sofern eine erhebliche Gewalteinwirkung erkennbar war, eine der beiden wesentlichen Teilursachen für den Gliedmaßenverlust $[2,10,16]$.

\section{Chronische Osteitis}

Sie ist meist durch das Auftreten von sezernierenden Fisteln und Sequestern gekennzeichnet. Im Verlauf kann der klinische Befund zwischen vollkommen blanden Weichteilen, kaum sezernierenden Fisteln und großflächigen Ulzera variieren. Die Fistelung ist ein „Selbstheilungsversuch“ des Körpers, wobei die eitrige Infektion die Oberfläche erreicht hat und sich der vorher allmählich im eigentlichen Osteitisherd angesammelte Eiter entleeren kann. Dadurch ist die Gefahr einer allgemeinen Infektausbreitung über den Lymph- oder Blutweg (Sepsis) vorerst gebannt.

Eine Fistelung ist keine Voraussetzung für das Vorhandensein einer Osteitis; insbesondere bei guter systemischer und lokaler Abwehrlage und Antibiotikatherapie kann der Weichteilbefund relativ unauffällig ausfallen.

Ohne operative Intervention kommt es nur selten zur Infektberuhigung. Die Grundlagen der chirurgischen Therapie bestehen in:

- der vollständigen Materialentfernung und ggf. externen Stabilisierung,

- Débridement,

- Sequestrektomie und

- Einlage von Antibiotikaträgern.

Anschließend müssen die Weichteile, häufig durch aufwändige Lappenplastiken, rekonstruiert werden. Erst dann kann der Knochendefekt durch unterschiedliche Techniken entsprechend der Defektgröße von Spongiosaplastiken über gefäßgestielten Knochenspänen bis hin zur Kallusdistraktion wieder aufgebaut werden.

Dieser Behandlungsverlauf ist äußerst langwierig, dauert oft über mehrere Monate bis Jahre an und ist durch häufige Operationen, die teilweise mit Komplikationen und Rückschlägen behaftet sind, gekennzeichnet. Die Erfolgsrate der In-
Trauma Berufskrankh 2009 · 11 [Suppl 2]:

222-228

DOI 10.1007/s10039-008-1445-2

(C) Springer Medizin Verlag 2008

M. Diefenbeck · R. Beickert · G.O. Hofmann

\section{Begutachtung der Osteitis}

\section{Zusammenfassung}

In der unfallchirurgisch-orthopädischen Begutachtung von Infektionen an Knochen und Gelenken steht fast ausschließlich die chronische exogene Osteitis als Folge einer Verletzung im Mittelpunkt. Die hämatogene Osteomyelitis im Zusammenhang mit einer Verletzung ist in Europa eine außerordentliche Seltenheit. Entscheidend für die Einschätzung der MdE (Minderung der Erwerbsfähigkeit) sind die aktuelle Funktionseinbuße und der klinische Befund. Die Funktionsminderung wird entsprechend den Grundsätzen der Begutachtung von Unfallfolgen erhoben. Der klinische Befund muss die Aktivität der Osteitis, also insbesondere das Vorliegen einer sezernierenden Fistel, instabiler Narben oder eines frakturgefährdeten Knochen beschreiben. Die Kombination beider Untersuchungen legt die Höhe der MdE bzw. der Gliedertaxe fest.

\section{Schlüsselwörter}

Osteitis · Begutachtung · Unfallversicherung . Minderung der Erwerbsfähigkeit · Gliedertaxe

\section{Expert opinion on posttraumatic osteomyelitis}

\section{Abstract}

Chronic posttraumatic osteomyelitis remains a major topic of expert discussion concerning infections of the bones and joints. Trauma-induced haematogenous osteomyelitis is rare in Europe. The two main determinants for the degree of disability are the degree of functional loss and the clinical examination. The degree of functional loss is analysed according to the standards of medical opinion in orthopaedic and trauma surgery. The clinical examination should address the activity of the osteomyelitis, i.e. the existence of a draining sinus. The combination of both defines the degree of disability and thus the compensation.

\section{Keywords}

Osteomyelitis - Expert opinion - Accident insurance $\cdot$ Degree of disability $\cdot$ Compensation 


\section{Sozio-ökonomische "Resistenz"-Entwicklung}

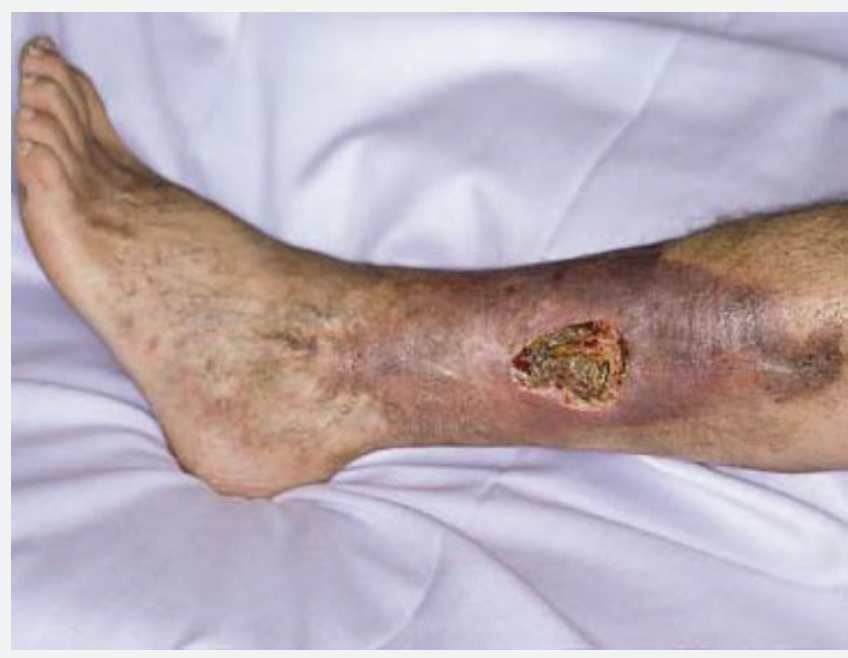

Abb. $1 \varangle$ Histologisch gesichertes Narbenkarzinom (Plattenepithelkarzinom)

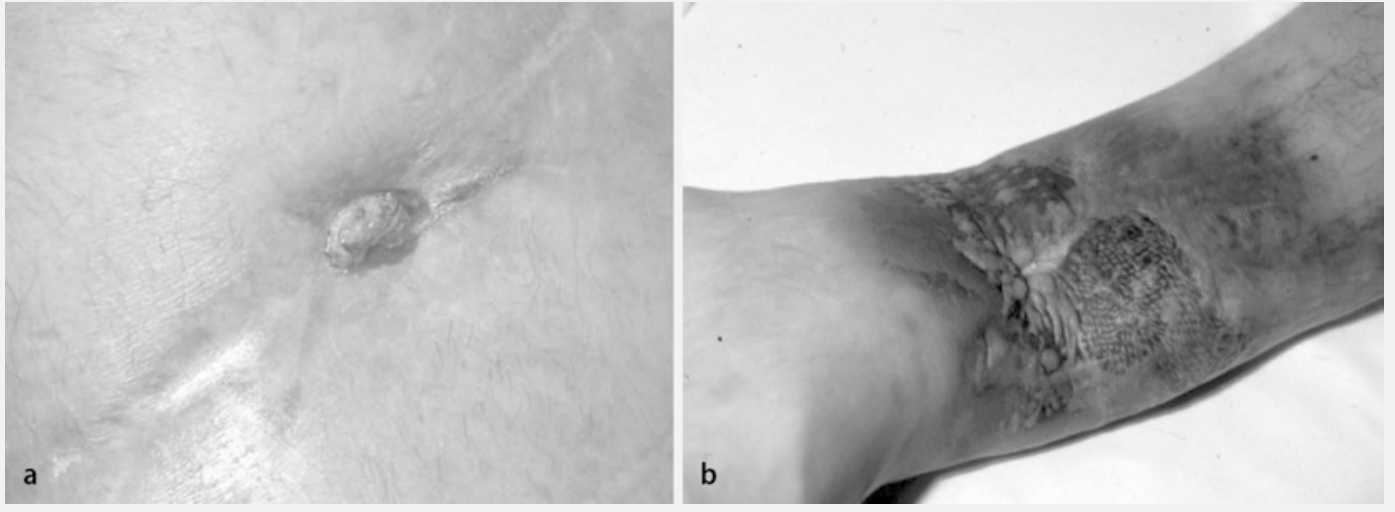

Abb. $2<$ Unterschiedliche Haut- und Weichteilbefunde bei chronischer Osteitis, a sezernierende Fistel, b Narbenplatte nach Weichteilrekonstruktion durch Lappenplastik

fektberuhigung wird unterschiedlich angegeben. Sie liegt in der Literatur in etwa zwischen 90 und 94\% [1, 11]; im klinischen Alltag scheint die Rezidivrate jedoch deutlich höher zu sein und wird von anderen Autoren auf 15-80\% geschätzt [9].

Das „Wiederaufflammen“ der Osteitis, auch als Rezidiv oder Exazerbation der scheinbar ruhenden oder beruhigten Osteitis bezeichnet, kann durch eine Änderung der Abwehrlage (Allgemeininfektionen, konsumierende Erkrankungen, Erstmanifestation eines Diabetes mellitus oder einer pAVK), durch ein Sekundärtrauma oder auch spontan auftreten. Als weitere Folgen einer Osteitis können Spontanfrakturen bei ausgedehnten Osteolysen durch den Infekt oder nach Knochenrekonstruktionen mit geringerer Stabilität („Sprödbrüche“) vorkommen.

\section{Kausalität}

Fragen zur Kausalität der chronischen Osteitis tauchen dann auf, wenn der zeitliche Zusammenhang zwischen der Verletzung und dem Auftreten der Entzün- dungszeichen nicht stimmig ist. Die teilweise in der Gutachtenliteratur noch vertretene Auffassung, ein ursächlicher $\mathrm{Zu}$ sammenhang zwischen Unfall und chronischer Osteitis sei abzulehnen, wenn die klinischen Symptome einer posttraumatischen Osteitis (Schmerz, Überwärmung, Rötung, Funktionseinschränkung) erst nach Ablauf eines Monats auftreten [16], ist nicht haltbar. Genau dieser Sachverhalt ist ja die Definition der chronischen Osteitis (s. oben). Das verzögerte Auftreten von Symptomen ist z. B. zu erwarten bei einer Kontamination durch eine offene Fraktur bei guter allgemeiner und lokaler Abwehrlage, stabiler Osteosynthese und mehrtägiger oder gar mehrwöchiger Antibiotikagabe. Somit ist auch die Forderung nach „Brückensymptomen“ (Hämatom, Schürfwunden usw.) innerhalb der ersten 4 Wochen nicht gerechtfertigt. Nur wenn erkennbar keine Knochenbruchverletzung bestand, eine primär bestehende erhebliche Weichteilquetschung/Weichteilwunde zeitgerecht abheilte und erst nach 1 Monat die ersten klinischen Symp- tome einer Osteomyelitis auftreten, sind Zweifel am kausalen Zusammenhang gerechtfertigt, auch wenn der Erstmanifestationsort der eitrigen Knochenentzündung mit dem der Gewaltentwicklung übereinstimmt. Lag eine frische Knochenbruchverletzung vor, die mit einem erheblichen Weichteilschaden verbunden war und wurde nicht operativ behandelt, ist eine später auftretende Osteitis posttraumatisch, wenn typische exogene Eitererreger vorliegen, die sich üblicherweise nicht hämatogen ausbreiten, auch wenn sich die ersten Symptome der Infektion erst nach mehreren Wochen einstellen.

Zunehmend spielen Folgeschäden im sozialen und psychischen Bereich bei der Begutachtung der chronischen Osteitis eine ernst zu nehmende Rolle. Die oft langjährige Behandlung mit mehrmonatigen Krankenhausaufenthalten kann zu Störungen des Ehe- und Familienlebens, zu sozialen Spannungen im Umfeld des Patienten bis hin zu Persönlichkeitsstörungen, Alkoholkrankheit und Depression führen. Diese Veränderungen richtig zu beurtei- 
len und gutachterlich zu bewerten, kann außerordentlich schwierig sein. Allerdings wird nach aktueller Auffassung der Deutschen Gesellschaft für ärztliche Psychotherapie keine MdE bei chronischer Osteitis auf psychologischem Fachgebiet gewährt.

\section{Komplikationen}

Da die operative Behandlung der chronischen Osteitis langwierig und kompliziert ist, kann es gerade hier zum Auftreten von Komplikationen kommen. Zu erwähnen sind:

- Pin-Track-Infektionen bei der langzeitigen Anwendung von Fixateur-externe-Systemen,

- unzureichende Knochenneubildung bis hin zu Pseudarthrosen,

- ausgedehnte Skelettentkalkung,

- chronische Schwellungszustände der unteren Extremitäten nach Kompromittierung des Lymphsystems und

- eine ausgedehnte Narbenbildung.

2 spezifische Komplikationen der chronischen Osteitis sind:

- die Entstehung einer Amyloidose und

- das Auftreten eines Fistelkarzinoms.

Amyloidose. Es handelt sich um eine generalisierte oder lokalisierte extrazelluläre Proteinablagerung im Interstitium verschiedener Organe in der Umgebung von Retikulinfasern und Basalmembranen [8]. Das Amyloid wird von Plasmazellklonen gebildet, die im Rahmen der Infektabwehr entstehen [13]. Betroffene Organe vergrößern und verhärten sich speckartig („Speckleber“, „Speckmilz“, „Sagomilz“). Je nach Organbefall kann eine Leber-, Nieren- oder Herzinsuffizienz resultieren.

Die Diagnosestellung erfolgt durch eine endoskopische Darmbiopsie; die Klärung der Kausalität bei der langjährig bestehenden chronischen Osteitis bereitet keine Schwierigkeiten.

Zur Einschätzung der Gesamt-MdE durch die Amyloidosefolgen wird ein $\mathrm{Zu}$ satzgutachten auf internistischem Fachgebiet empfohlen.

Fistel- und Narbenkarzinome. Sie treten auf dem Boden einer chronischen Osteitis in etwa 0,21-1,5\% der Fälle auf (• Abb. 1)

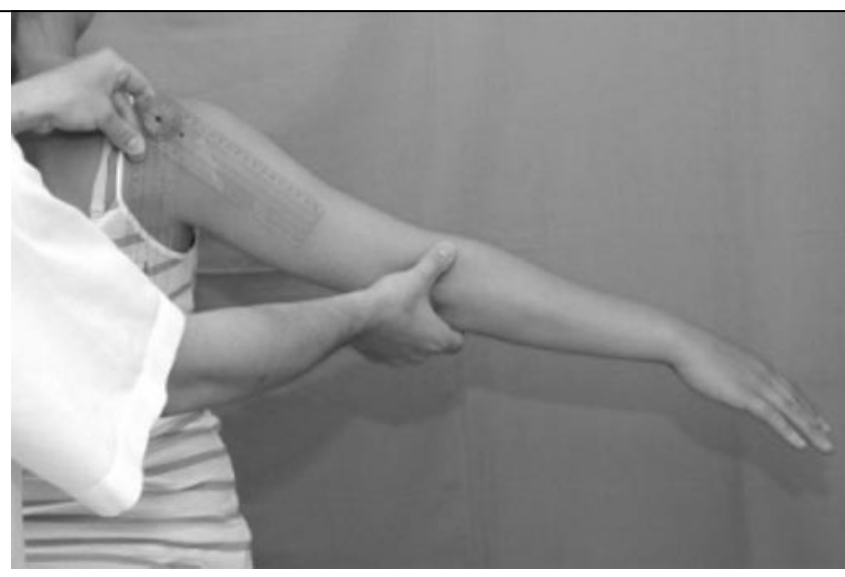

[6]. Die Zeitspanne zwischen Erstverletzung und Manifestation des Karzinoms ist sehr lange; sie beträgt in etwa 30 Jahre [6]. Histologisch liegen in der Regel Plattenepithelkarzinome oder Basalzellkarzinome vor. Die betroffene Extremität muss in der Regel amputiert werden. Gutachterlich ist auch hier die Kausalitätsklärung unproblematisch.

\section{Gelenkinfekt/Gelenkbeteiligung}

Da eine offene Gelenkverletzung im Vergleich zur offenen Fraktur deutlich seltener auftritt, ist auch die Inzidenz der posttraumatischen Gelenkinfektion durch das direkte Eindringen der Bakterien über eine Gelenkeröffnung relativ niedrig. Häufiger kommt es zu einer Gelenkinfektion bei bestehender Osteitis durch die Ausbreitung der Erreger über die Weichteile ins Gelenk. Bei Gelenkpunktionen, Arthroskopien oder offenen Gelenkeingriffen kann iatrogen ein Infekt ausgelöst werden.

Wichtig für die Begutachtung ist, dass eine Gelenkinfektion zu irreversiblen Knorpelschäden führen kann, die dann möglicherweise in einer postinfektiösen Arthritis enden. Diese Gelenkveränderungen können eine ausgeprägte Bewegungs- und somit Funktionseinschränkung bis hin zur spontanen Versteifung (Ankylose) des Gelenks zur Folge haben.

Bei schmerzhafter Bewegungseinschränkung bestehen therapeutisch je nach betroffenem Gelenk die Möglichkeiten des endoprothetischen Gelenkersatzes oder der Arthrodese.

\section{Gutachterliche Untersuchung}

Die Begutachtung hat unabhängig davon, auf welcher Rechtsgrundlage sie erfolgt, immer mehrere Aspekte:

- Darstellung des bisherigen Verlaufs

- Beschreibung des Istzustands

- Wertung der Unfallfolgen

- Skizzierung der möglichen zukünftigen Entwicklung.

Bei der Begutachtung der Osteitis gelten die gleichen Regeln wie bei anderer Unfallfolgen. Die gutachterliche Untersuchung gliedert sich in die Beschreibung des Allgemeinzustandes einschließlich Körpergröße und -gewicht und des örtlichen Befundes. Dabei kann man sich bei der Gesetzlichen sowie der Privaten Unfallversicherung auf den verletzten Körperteil und dessen Vergleich zur gesunden Gegenseite beschränken; eine Ganzkörperuntersuchung ist nicht notwendig.

\section{Istzustand}

\section{Klinik}

Die Untersuchung sollte schematisiert erfolgen, um die Vollständigkeit und Reproduzierbarkeit zu gewährleisten. Der örtliche Befund umfasst alle sichtbaren Veränderungen und alles, was sich tastend und berührend beschreiben lässt.

Unter dem Punkt „Betrachtung“ sollte bei der Osteitis besonderer Wert auf die Beschreibung von Narben, Fistelungen (inklusive Sekretart und -menge) und Kontrakturen gelegt werden (• Abb. 2). Zusätzlich ist auf Schwellungen von Gelenken und auf die Verschmächtigung von Muskelpartien zu achten. 


\section{Sozio-ökonomische "Resistenz"-Entwicklung}
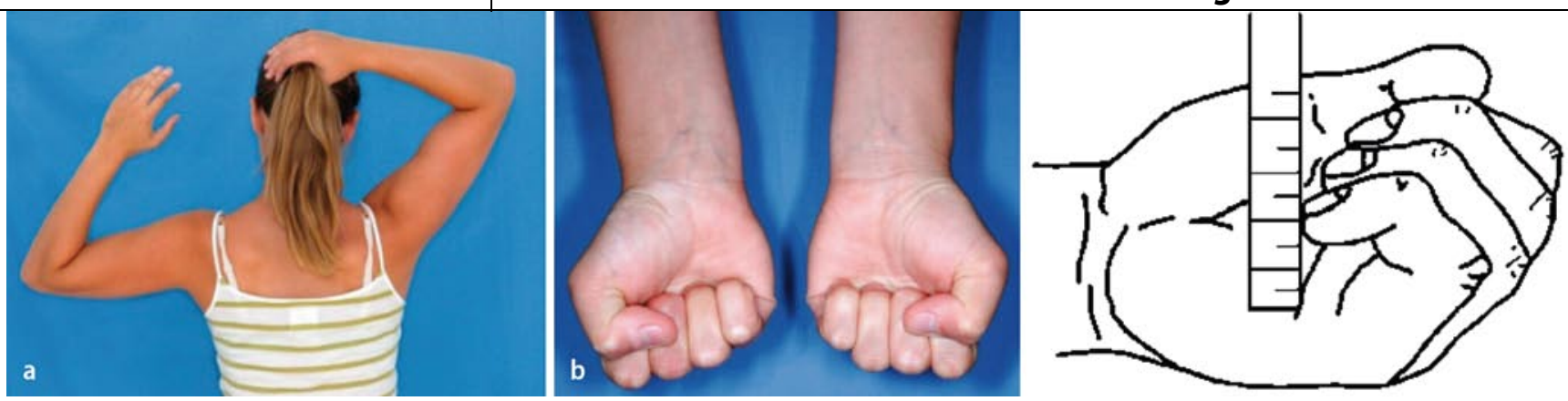

Abb. 4 \ Funktionstests zur Überprüfung der Gebrauchsfähigkeit der oberen Gliedmaßen, a Nackengriff, b Faustschluss

Bei der „Betastung“ sollten Verhärtungen durch Narbenplatten oder Kontrakturen, druckschmerzhafte Areale, verhärtete Lymphknoten und ödematöse Schwellungen dokumentiert werden.

Um Funktionsstörungen qualitativ und quantitativ zu erfassen, muss der entsprechende Messbogen (obere/untere Gliedmaßen; Wirbelsäule) vollständig ausgefüllt werden ( $\mathbf{D}$ Abb. 3). Er wird auch für die Verlaufskontrolle bei Nachuntersuchungen benötigt.

Abschließend sollte beschrieben werden, wie die betroffene Extremität zum Gebrauch eingesetzt werden kann. An der oberen Extremität wären dies z. B. Faustschluss und Fingerstreckung, Halteversuche, Krafttests und Armgriffe (Überkopfgriff, Nackengriff und Schürzenbindegriff) (• Abb.4). An der unteren Extremität werden das Gangbild, der Zehenspitzen- und Fersengang, der 1-BeinStand und z. B. das Ausführen einer Kniebeuge beschrieben.

Im Zeitalter der digitalen Fotografie hat es sich als vorteilhaft erwiesen, die Hautbefunde und die wesentlichen Funktionsstörungen $\mathrm{zu}$ fotografieren und die Bilder als Ausdruck dem Gutachten beizulegen.

\section{Bildgebende Diagnostik}

Aktuelle Röntgenaufnahmen des verletzten Gliedmaßenabschnitts sind unerlässlich zur Beurteilung der Stabilität des Knochens, dem Vorhandensein von Osteolysen oder Sequestern und des Kalksalzgehalts. Die angrenzenden Gelenke sollten nur dann mit abgebildet werden, wenn die Osteitis gelenknah abläuft. Ansonsten sollten Standardeinstellungen für die Beurteilung der Gelenke gewählt werden.
Weitere bildgebende Untersuchungen sind nur angebracht, falls die Notwenigkeit einer Wiederaufnahme der Behandlung besteht, Fragen zur Kausalität beantwortet werden müssen oder wenn nicht sicher ist, ob überhaupt eine chronische Osteitis vorliegt.

\section{Labordiagnostik}

Das C-reaktive Protein kann sensibel, aber sehr unspezifisch eine akute und chronische Entzündung anzeigen. Ähnlich verhält es sich mit der Leukozytenzahl. Das einmalig bei einer Begutachtung ermittelte Ergebnis ist meist ohne Wert, der Vergleich mit Vorbefunden kann jedoch einen Hinweis über den Grad der Aktivität der Osteitis, also ob ein „ruhendes“ oder ein „im Aufflammen befindliches“ Stadium vorliegt, geben.

\section{Wertung}

\section{Gesetzliche Unfallversicherung}

Eine chronische Osteitis kann z. B. an den oberen Gliedmaßen lokal so begrenzt sein, dass beim Fehlen von Beschwerden und funktionellen Einschränkungen die MdE bei 10\% oder darunter liegt. Derart günstige Verläufe sind jedoch die Ausnahme.

Bei der häufigsten Lokalisation, der chronischen Osteitis des Unterschenkels, ist im Regelfall nicht nur die Behandlung sehr langwierig, sondern auch die berufliche Wiedereingliederung erschwert, sodass die Rentenversicherungsträger häufig eine zeitlich begrenzte Erwerbsunfähigkeit feststellen müssen. Im Rechtsbereich der Gesetzlichen Unfallversicherung ist dann erstmals der Zustand nach Ablauf der 78 . Woche nach dem Unfall zu beurteilen. Häufig befinden sich die Patienten zu diesem Zeitpunkt immer noch in Behandlung, da der schematisch gegliederte
Behandlungsablauf - Infektberuhigung Weichteildeckung - Knochendefektaufbau - berufliche Wiedereingliederung - noch nicht abgeschlossen ist. Ein Verletzter, an dessen Bein noch ein Fixateur externe angebracht ist und der zur Teilbelastung auf den Dauergebrauch zweier Gehstützen angewiesen ist, ist in dieser Behandlungsphase schlechter gestellt als ein Unterschenkelamputierter mit gut funktionierender Prothese (• Abb.5). In diesem Beispiel wäre die MdE im ersten Rentengutachten so abzustufen, wie im Regelfall die weitere Entwicklung zu erwarten ist. Zur Kontrolle sollte schon nach 6 Monaten eine Rentenüberprüfung anberaumt werden. Die Höhe der MdE richtet sich nach den Funktionseinbußen und liegt zunächst bei oder über 50\% bis zur Abnahme des Fixateur externe und zur Wiederbelastung des Beins. Muss im weiteren Verlauf ein Stützapparat getragen werden, was nach der Überbrückung von Knochendefekten regelhaft empfohlen wird, liegt die MdE bei etwa $40 \%$. Kommt es zur knöchernen Konsolidierung, Abnahme des Stützapparats und Weglassen der Gehstützen liegt die MdE bei 30\% und damit erstmals unter Werten der Unterschenkelamputation. In dieser MdE sind Muskelminderungen am Oberschenkel und geringgradige Bewegungseinschränkungen im Knie und Sprunggelenk mit einer Spitzfußstellung bis $10^{\circ}$ enthalten. Sind darüber hinausgehende Funktionsstörungen am Knie (Streckdefizit $>10^{\circ}$ ) und/oder am Sprunggelenk und Fuß (Einsteifung oder Versteifung des oberen/unteren Sprunggelenks, Krallenzehenbildung usw.) vorhanden, liegt bei der ersten, vorläufigen Einschätzung der Rente die MdE zwischen 30 und $40 \%$, bei zusätzlichen neurologischen Störungen noch darüber. Grundlage hierfür 
sind die Einschätzungsempfehlungen bei der gestörten Knochenbruchheilung am Unterschenkel [16].

Bis ein stabiler Zustand eingetreten ist und die Rente auf unbestimmte Zeit festgelegt werden kann, orientiert sich bei bestehender chronischer Osteitis des Unterschenkels oder nach Infektberuhigung durch Resektion und Osteoneogenese die MdE ausschließlich an den Funktionseinbußen an den benachbarten Gelenken, der Stabilität der ehemaligen Fraktur oder des neu gebildeten Knochens und der Aktivität der Osteitis. Im Rahmen der Begutachtung von Gelenkinfektionen mit nachfolgender Arthrodese empfahl Roesgen [14], MdE Aufschläge zu gewähren:

- 10-20\% für eine sezernierende Fistel,

- $10 \%$ für die Notwendigkeit eines

Schutzverbandes und

- $10 \%$ für eine ungünstige Narbenbildung.

Dieser Vorschlag sollte auch für die aktive chronische Osteitis umgesetzt werden. Die ruhende Osteitis ist dabei mit einer chirurgisch sanierten Osteitis gleichzusetzten, deren MdE am Unterschenkel, sofern keine weiteren neurologischen Defizite oder Durchblutungsstörungen bestehen, immer unter $40 \%$ liegen wird. Die aktive, fistelnde Osteitis dagegen wird - entsprechende funktionelle Einschränkungen vorausgesetzt - auch unter Dauerrentengesichtspunkten eine MdE erreichen, die einem Verlust des Beins im Unterschenkel gleichkommt (MdE von 40\%).

Nachdem ohnehin empfohlen wird, alle Versicherten mit einer chronischen, fistelnden Osteitis mindestens in Jahresabständen im Rahmen von Heilverfahrenskontrollen nachzuuntersuchen, ist gewährleistet, dass auf Änderungen des Aktivitätsgrads der Osteitis reagiert werden kann.

\section{Private Unfallversicherung}

Laut den Allgemeinen Unfallversicherungsbedingungen (AUB) der privaten Unfallversicherer ist die Versicherungssumme spätestens zum Ende des 3. Jahres nach dem Unfall auszuzahlen. Es ist möglich, dass die Behandlung zu diesem Zeitpunkt noch gar nicht abgeschlossen ist. Gerade bei der chronischen Osteitis kann dann ein Zustandsbild bestehen, in dem der Versicherte schlechter gestellt ist als bei einem Gliedmaßenverlust. Dennoch darf die 3-Jahres-Frist nicht überschritten werden: Die Versicherungssumme muss zur Auszahlung kommen, auch wenn noch eine wesentliche Besserung zu erwarten ist.

Die Höhe des Auszahlungsbetrags richtet sich nach der vertraglich vereinbarten Versicherungssumme und den funktionellen Einbußen der betroffenen Gliedmaßen. Hieraus wird die so genannte Gliedertaxe abgeleitet. Die funktionellen Einbußen werden genauso ermittelt und beschrieben wie in den anderen Versicherungszweigen, dann aber als Gebrauchsbeeinträchtigung der betroffenen Gliedmaße bezogen auf ein gesundes Bein/Arm ermittelt und in Bruchteilen der normalen Gebrauchsfähigkeit abstrakt gebildet.

Nachdem für den Verlust im Oberschenkel 70\% der Versicherungssumme ausgezahlt werden, ist es praktikabel, die Gebrauchsbeeinträchtigung eines Beines in Siebteln darzustellen, weil dann z. B. nach einem Arbeitsunfall oder bei der Feststellung der GdB durch das Versorgungsamt auch nach außen hin zum Ausdruck kommt, dass der Unfallfolgezustand immer gleich hoch bewertet wird. Man erspart sich und dem Versicherten damit unnötige Rückfragen und Streitereien.

Eine chronische Osteitis des Unterschenkels mit Fistelbildung und geringen Funktionseinschränkungen an den Fuß- und Zehengelenken wird maximal mit 3/7 Beinwert eingestuft. Eine ruhende oder erfolgreich chirurgisch beruhigte Osteitis mit freier Funktion des Knie- und Fußgelenks wird mit maximal 1/7 eingestuft.

\section{Einschätzungsempfehlungen}

Anhand einer ruhenden und einer aktiven Osteitis der oberen Gliedmaßen werden für die Gesetzliche (MdE) und für die Private Unfallversicherung (Gebrauchsbeeinträchtigung) folgende Empfehlungen gegeben:

Obere Gliedmaßen, ruhende Osteitis. Die MdE bzw. die Gebrauchsbeeinträchtigung richtet sich nach den Funktionseinbußen [16].

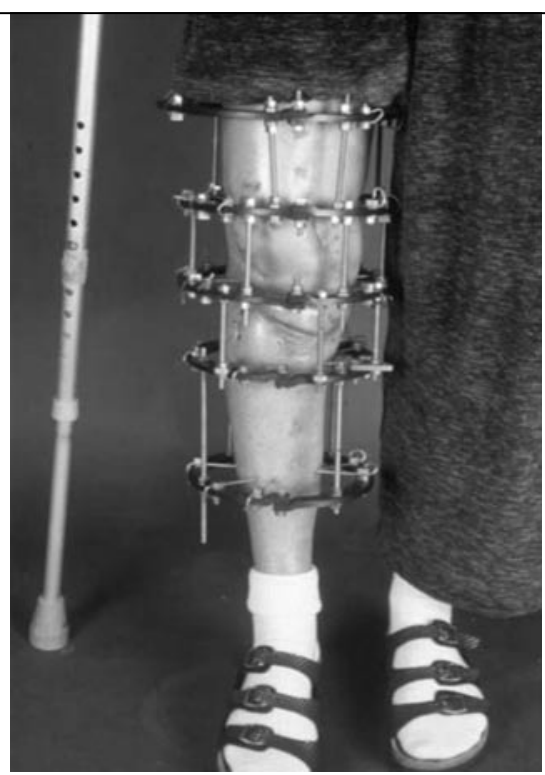

Abb. $5 \Delta$ Patient nach Rekonstruktion eines Knochen- und Weichteildefekts nach Beruhigung einer chronischen Osteitis, noch einliegender llisarov-Ringfixateur, Patient bei einer Teilbelastung rechts von $20 \mathrm{~kg}$ auf 2 Unterarmgehstützen angewiesen

Beispiele. Zustand nach chirurgischer Infektberuhigung nach proximaler $\mathrm{Hu}$ merusfraktur mit Schultersteife, Hebung aktiv unter $90^{\circ}$, Außenrotation eingeschränkt:

- $M d E_{30 \%}$

- Beeinträchtigung 3/7.

Zustand nach beruhigter akuter Osteitis nach offener Olekranonfraktur mit Ellenbogenteilsteife im Umfang O-30-120 ${ }^{\circ}$ :

- MdE $10 \%$

- Beeinträchtigung $1 / 7$.

Aktive Osteitis. Die MdE richtet sich nach den Funktionseinbußen plus einem Aufschlag für chronische Fisteln je nach Ausmaß von 10-20\%, Beeinträchtigung $1 / 7-2 / 7$.

Beispiele. Chronische Osteitis nach proximaler Humerusfraktur mit Schultersteife, Hebung aktiv unter $90^{\circ}$, Außenrotation eingeschränkt, chronische Fistel mit wenig seröser Sekretion:

- MdE: 30\%, plus Aufschlag von 10\%, entspricht $40 \%$

- Gebrauchsbeeinträchtigung 3/7, plus Aufschlag 1/7, entspricht 4/7

Chronische Osteitis nach offener Olekranonfraktur mit Ellenbogenteilsteife im 
Umfang $0-30-120^{\circ}$ und massiv sezernierender Fistel, übel riechendes Sekret mit der Notwendigkeit, mehrfach täglich den Verband zu wechseln:

- MdE: 10\%, plus Aufschlag 20\%, entspricht $30 \%$

- Gebrauchsbeeinträchtigung 1/7, plus Aufschlag 2/7, entspricht 3/7.

\section{Fazit für die Praxis}

Die Begutachtung der chronischen Osteitis muss sich nur in seltenen Fällen mit der Kausalitätsfrage befassen. Im Regelfall besteht sie in einer sorgfältigen klinischen Untersuchung mit zusammenfassender Beschreibung der funktionellen Einbußen an der betroffenen Extremität. Dabei muss die Frage geklärt werden, ob zum Zeitpunkt der Begutachtung eine aktive „fistelnde" Osteitis besteht oder ein Ruhezustand, z. B. nach chirurgischer Infektberuhigung, vorliegt. Anschließend erfolgt die Wertung des Körperschadens nach den vorgegebenen Regeln der Funktionsbegutachtung, die sich an objektiven Tatsachen orientiert.

\section{Korrespondenzadresse}

\section{Dr. M. Diefenbeck}

Klinik für Unfall-, Hand- und
Wiederherstellungschirurgie,
Friedrich-Schiller-Universität
Jena
Erlanger Allee 101,07747 Jena
Michael.Diefenbeck@med.uni-
jena.de

Interessenkonflikt. Der korrespondierende Autor gibt an, dass kein Interessenkonflikt besteht.

\section{Literatur}

1. Ascherl R, Stemberger A, Lechner F et al. (1986) Treatment of chronic osteomyelitis with a collagen-antibiotic compound - preliminary report. Unfallchirurgie 12: 125-127

2. Böhm HJ, Kortmann HR (2002) Besonderheiten bei der Begutachtung der Osteitis. Trauma Berufskrankh 4: 340-343

3. Boxma H, Broekhuizen T, Patka P et al. (1996) Randomised controlled trial of single-dose antibiotic prophylaxis in surgical treatment of closed fractures: the Dutch trauma trial. Lancet 347: 11331137

4. Carsenti-Etesse H, Doyon F, Desplaces N et al. (1999) Epidemiology of bacterial infection during management of open leg fractures. Eur J Clin Microbiol Infect Dis 18: 315-323
5. Espehaug B, Engesaeter LB, Vollset SE et al. (1997) Antibiotic prophylaxis in total hip arthroplasty. Review of 10,905 primary cemented total hip replacements reported to the Norwegian arthroplasty register, 1987 to 1995. J Bone Joint Surg Br 79: 590-595

6. Faschingbauer M, Jürgens $C$, Schmidt HGK et al. (1995) Ergebnisse der Behandlung von Narbenund Fistelkarzinomen. Chirurg 66: 1141-1145

7. Gentry LO (1988) Osteomyelitis: options for diagnosis and management. J Antimicrob Chemother [Suppl C] 21: 115-128

8. Herold G (Hrsg) (2007) Innere Medizin. Selbstverlag, Köln

9. Hofmann GO (Hrsg) (2004) Chronische Osteitis. Infektionen der Knochen und Gelenke. Urban \& Fischer, München Jena

10. Jostkleigrewe F, Turban KL (1986) Die richtige Einschätzung der MdE in der gesetzlichen Unfallversicherung bei exogener Osteomyelitis. In: Hierholzer G, Ludolph E (Hrsg) Gutachtenkolloquium 1. Springer, Berlin Heidelberg New York

11. Klemm K (1983) Local treatment of bone and soft tissue infections using antibiotic release carriers. In: Kayser FH, Machka K, Wieczorek L et al. (eds) Proceedings of the 13th International Congress of Chemotherapy, Vienna. SS 7.23/1, SS 7.23/2, Part 43

12. Kutscha-Lissberg F, Hebler U, Kälicke T et al. (2002) Inzidenz, Diagnose, Manifestations- und Verlaufsformen der Osteitis. Trauma Berufskrankh 4: 297

13. Riede UN, Schaefer HE (2006) Allgemeine und spezielle Pathologie. Thieme, Stuttgart New York

14. Roesgen M (2001) Die Gelenkinfektion und ihre ärztliche Begutachtung. In: Hierholzer G, Kortmann HR, Kuntze G et al. (Hrsg) Gutachtenkolloquium 15. Springer, Berlin Heidelberg New York

15. Schmidt HGK, Hadler D, Wurm M et al. (2003) Therapie der Infekt-/Defektpseudarthrosen der unteren Extremitäten. Trauma Berufskrankh [Suppl 2] 5: S318-S327

16. Schönberger A, Mehrtens G, Valentin H (2003) Arbeitsunfall und Berufskrankheit, 7. Aufl. Schmidt, Berlin 